Digital Press Social Sciences and Humanities

Le modèle d'apprentissage de la production orale à travers Skype basé sur la culture

Amalia Saleh, Ninuk Lustyantie and Evi Rosyani Dewi

Proceeding of Conférence internationale sur le français 2018

Joesana Tjahjani, Merry Andriani, Sajarwa, Wening Udasmoro (eds) 


\title{
Le modèle d'apprentissage de la production orale à travers Skype basé sur la culture
}

\author{
Amalia Saleh*, Ninuk Lustyantie et Evi Rosyani Dewi \\ Universitas Negeri Jakarta, Jakarta, Indonesia \\ *e-mail : amalia284@yahoo.fr
}

\section{Résumé}

A l'université, on a besoin d'une stratégie d'apprentissage du français parce qu'on n'apprend pas seulement le français mais aussi les cours en commun. Donc, on n'a pas assez le temps pour pratiquer la conversation dans la classe. C'est pour cela que les professeurs doivent choisir un média social pertinent comme Skype. Ce média permet d'accéder aux étudiants gratuitement à longue distance et à n'importe quel moment. La recherche dont le titre est le modèle d'apprentissage de la production orale à travers Skype basé sur la culture a pour but d'analyser les activités de production orale du manuel et de les planifier en se basant sur la culture dont on a besoin. On a observé le manuel l'Alter Ego+1 que les étudiants utilisent et on a noté quels thèmes existaient, par exemple : l'identité, lieu (indiquer le chemin), la passion et le loisir, les activités de tous les jours, la famille et la vie, le voyage, la gastronomie et la mode. Cette recherche qui utilise une approche qualificative et une méthode de la recherche du développement est un plan pour rechercher et développer le modèle d'apprentissage. Et après, on fait le prototype du modèle apprentissage de la production orale à travers Skype basé sur la culture. Voici quelques étapes avant de produire ce modèle : analyser le manuel, développer la matière de la production orale, valider la matière. Les étapes de cette recherche utilisent le modèle de Borg et Gall.

\section{Mots-clés}

français, modèle d'apprentissage, production orale, Skype

\begin{abstract}
At university, we need a strategy for learning French because we are not only learning French but also the courses in common. So we don't have enough time to practice the conversation in the classroom. This is why teachers have to choose a relevant social media such as skype. This medium allows free access to students at long distance and at any time. Research called the Culture-Based Skype Oral Production Learning Model aims to analyze the manual's oral production activities and plan them based on the culture needed. We observed the Alter Ego+1 manual that students use and we noted which themes existed, for example: identity, place (point the way), passion and leisure, everyday activities, family and life, travel, gastronomy and fashion. This research, which uses a qualitative approach and a method of research and development, is a plan for researching and developing the learning model. And then we make the prototype of the learning model of oral production through Skype based on culture. Here are some steps before producing this model: analyze the manual, develop the oral production material, validate the material. The steps in this search use the Borg and Call model.
\end{abstract}

\section{Keywords}

French, learning model, oral production, Skype

\section{Introduction}

Le processus d'apprentissage est un système qui a des politiques et des stratégies pour améliorer la qualité de l'éducation. Afin d'améliorer la qualité de l'éducation, c'est nécessaire pour analyser chaque élément qui affecte la qualité de l'éducation. L'un des éléments qui influe le processus d'enseignement est 
l'enseignant parce que l'enseignant est la mise en œuvre réussie du processus. La première est la capacité de l'enseignant à concevoir une stratégie d'apprentissage qui convient à l'objectif.

Pour implémenter une stratégie, l'enseignant est tenu de changer le paradigme de l'enseignement. Ceci est très important car avec le développement des technologies de l'information, les enseignants peuvent utiliser l'information et la technologie de communication comme une stratégie d'apprentissage. Grâce à TIC, l'apprentissage des processus qui était auparavant uniquement dans la salle de classe, peut maintenant être hors de la classe, même en dehors de la ville ou l'État. L'enseignement à distance en utilisant l'internet, des enseignants peuvent facer aux téléconférences, les enseignants et élèves communiquent en temps réel.

L'université d'état de Jakarta, actuellement n'utilise pas l'enseignement à distance comme Skype. Prodi de français utilise le livre d'Alter Ego par Berthet, et al. Cet ouvrage a été utilisé pour les 4 compétences, les compétences de lecture, d'écoute, d'écriture et de parler. Pour l'expression orale, le processus d'apprentissage sert aux thèmes et aux expressions. Par exemple dans la première leçon, on parle de saluer, se présenter, présenter quelqu'un ou se renseigner sur l'identité de l'autre personne et ainsi de suite. Le livre se compose de 9 chapitres restant à accomplir en deux semestres et est livré avec un CDROM.

On utilise le système d'apprentissage pour la première année par l'enseignement des 4 compétences intégrées. Il n'y a aucune séparation, des cours, compréhension orale, compréhension écrite, production orale et production écrite. N'importe quel professeur plus tard mettrait l'accent sur le matériel d'apprentissage qu'il enseigne. L'apprentissage pour 4 compétences a 12 crédits par semestre. Pour la production orale, les activités de cet apprentissage sous la forme de coaching, la forme des structures de phrases, de jeux de rôles et d'autres activités. Avec un temps limité, chaque semestre, les étudiants doivent finir 5 unités du manuel de l'Alter Ego +1 et ils doivent connaitre les expressions, les actes de parole tous ce qu'il y a dans ce manuel. Le problème a été découvert quand un étudiant parlant qu'ils révèlent l'expression indonésienne et ensuite traduisent en langue française. Alors, certains étudiants ont de la difficulté de parler et en plus pour parler, on n'a pas seulement besoin de la structure mais aussi du contexte et de culture parce que tout cela joue un rôle important dans la conversation.

Pour atteindre cet objectif, on a besoin beaucoup de temps d'entrainer les étudiants à parler naturellement. En donnant les exercices et l'entrainement c'est la façon la plus pertinente mais comme on n'a pas beaucoup de temps donc on ne peut pas finir le programme, les professeurs doivent prolonger le temps. Avec le développement des technologies de l'information, l'enseignant et les apprenants peuvent servir au réseau de l'internet, c'est Skype, Skype peut fournir les enseignants qui veulent enseigner par de longues distances. Dans ce cas, les enseignants et les élèves peuvent communiquer en temps réel et n'importe où et n'importe quand, parce qu'ils peuvent communiquer en vidéo de Skype et en appels audio, échanger des messages, chatter et faire bien d'autres choses en utilisant le logiciel Skype sur les ordinateurs, les téléphones mobiles, tablettes et autres appareils.

\section{Méthode}

La méthode utilisée dans cette recherche est celle de l'approche culturelle et celle de recherche et développement. Les données de cette recherche sont les actes de paroles et les thèmes du manuel de l'Alter Ego+. Cette méthode a pour but de produire une certaine chose et valider son efficacité.

Pour le modèle d'apprentissage, cette recherche a utilisé la théorie de Borg et Gall (1983) a déclaré il y a des stratégies de mise en œuvre étapes : la recherche et développement, planification (Planning), la validation, le prototype.

Collection de recherche et d'information - la collection de données - le dessin de produit - la validation - le prototype.

\section{Résultat et discussion}

Après avoir analysé le manuel d'Inter Ego+en 1, on recherche les thèmes et les actes de paroles qui contiennent les aspects culturels français, on les interprète. Les aspects qu'on a trouvés, son identité, lieu (indiquer le chemin), passion et loisir, activités de tous les jours, famille et la vie, le voyage, gastronomie et mode.

L'identité est une caractéristique de deux ou de plusieurs qui sont différents par la façon de désignation et par le lieu. L'identité se montre par la salutation et aussi l'emploie du sujet" vous" et" tu". 
Le lieu de vie est un aspect significatif dans la vie des êtres humain. On peut voir le type de logement est différent. Si on habite dans une grande ville, le type de logement n'est pas pareil de celui à la campagne, par exemple maison, appartement, studio.

La passion et loisir sont l'aspect culturel et cela se voit par le hobby de quelqu'un, les français aiment bien lire quand ils ont le temps libre ou promènent leur chien.

Les activités de tous les jours se présentent par le réveil jusqu'au couche et aussi par les horaires d'ouverture et de la fermeture du magasin, du musée, etc.

La famille est très importante pour former notre caractère, on sent heureux, triste, on peut se donner, se comprendre etc. Le concept de la famille dans chaque pays est différé. Selon Samovar (2014, p. 65) il y a des différences types pour la famille par exemple, la polygamie, le concubinage, le mono parental, la mère non mariée. Tous cela se voit par les vocabulaires français et indonésien.

Le voyage est une des préférences des français. Ils aiment voyager dans ou hors de la France. Ils préparent avant le départ et étudient bien le lieu où ils vont. Ils préparent aussi ce qu'ils veulent y faire.

La gastronomie et la mode sont icones pour la France. Il y a beaucoup de cuisines ou les gâteaux français qui sont connus, par exemple steak, macaron, croissant, etc. et alors, il faudrait savoir aussi la recette et comment faire le gâteau.

Pour la mode aussi, c'est très connu par les couturiers par exemple, Coco Chanel, Christian Lacroix. On peut savoir comment s'habiller correctement, quelle couleur, quel tissu on utilise.

A partir des thèmes qu'on a trouvés, on planifie les étapes de faire le prototype de model apprentissage à travers le Skype.

Avant de planifier les étapes, on doit connaitre d'abord ce que c'est le modèle. Le modèle peut également être vu comme une tentative d'objective. Il y a certaines théories entre autres, selon Robbins, « un modèle est une abstraction de la réalité ; une représentation simplifiée d'un phénomène réel. » (1996, p. 25). Le but de cette définition d'un modèle est une représentation d'un phénomène qui existe dans le monde réel. La définition du modèle est également exprimée par Miarso (2011) le modèle est une représentation d'un processus sous forme de graphiques ou de récit, en montrant les éléments majeurs ainsi que sa structure.

Le modèle de l'apprentissage, on doit effectuer le matériel de l'apprentissage et trouver les médias convenables pour les apprenants parce que c'est utile le matériel d'apprentissage. Tagliante (1994, pp. 62-65) dit que le matériel d'apprentissage fait partie des principales composantes d'un outil d'apprentissage qui ont suivi plus tard avec les appuis techniques tels que des partisans ou des médias, une robinet radio, une télévision et ainsi de suite ainsi que de haut-parleurs original pour apprendre une langue étrangère.

Pour cette recherche, on utilise les étapes ou les procédés de recherche et développement Borg et Gall (1983). Il a déclaré qu'il y a une stratégie de mise en œuvre 10 étapes de la recherche et développement, couvrant :

1. Recherche et collecte de données (collection de recherche et d'information) ; sur la recherche et les données collecte, elle a été réalisée en analyse des besoins, l'étude et la littérature de recherche à petite échelle.

2. Planification (Planning) ; Au stade de la planification de capacité d'identification, elle fait requis pour l'exécution de la recherche, faire les grandes lignes des objectifs à atteindre, de créer une étape de conception ou de recherche et planifier la possibilité de tester dans une portée limitée.

3. Mise en développement de produits ou de projet (mettre en place une forme préliminaire du produit) ; Développement de produits inclut la préparation des matériaux, l'apprentissage et instruments d'évaluation de l'apprentissage.

4. Premiers essais sur le terrain (essais sur le terrain préliminaire) ; Les premiers essais sur le terrain ou les tests effectués sur un nombre limité de 6-12 le sujet. Lors des essais effectués formulaire question d'observation, des entrevues et la circulation. Le but est d'obtenir une évaluation qualitative du produit en cours d'élaboration.

5. Révision du produit principal (révision de produit principal) ; Révisions de produit majeur faites se fondant sur les constatations sur les premiers essais sur le terrain.

6. Les Tests principaux (Main essais sur le terrain) ; Cet essai est effectué sur un sujet de 30-100. Données quantitatives sur l'apparence des enseignants, avant et après l'utilisation du modèle recueillies. Les données obtenues, plus évalués et si possible aussi par rapport au groupe contrôle.

7. Affinement du produit opérationnel (révision produit opérationnel) ; Raffinement de produit opérationnel se fait basé sur conclusions lors de l'implémentation des essais importants sur le terrain. 
8. L'essai opérationnel (principe essais sur le terrain) ; Cet essai est effectué sur un sujet de 40-200. Analyses sont effectuées par le biais de l'instant, interview, observation.

9. Réalisation du produit final (révision du produit Final) ; Le raffinement se fait sur les résultats des essais opérationnels sur le terrain sur la base.

10.Désemination et mise en œuvre (diffusion et mise en œuvre) ; Générer des rapports sur les produits lors d'une réunion de professionnels et le publier dans un journal, en collaboration avec l'éditeur, surveiller la répartition de faire le contrôle de la qualité.

On va conclure que les stratégies pour cette recherche est : la recherche et développement, planification (Planning), la validation, le prototype

A partir de cela, on produit un produit matériel apprentissage production orale utilisant Skype pour atteindre l'objectif de l'apprentissage car le Skype est un programme basé sur l'internet pour tout le monde d'assurer la qualité des moyens de communication. Les utilisateurs de Skype peuvent parler à d'autres utilisateurs Skype gratuitement, entrer en contact avec les frais de téléphone classique (Skype Out), recevoir des appels de téléphones fixes (Skype In), ainsi que diverses fonctionnalités qui a évolué besoins de la communauté. Distance d'apprentissage elle-même, a subi une évolution et très efficace. Selon Deborah Barreau après ses études au cours des années (2000, pp. 79-93), a montré que le téléapprentissage sur plusieurs sujets, y compris les langues, se sont avérés capables d'augmenter la puissance de concentration et de créer des étudiant devenant davantage axé sur le sujet d'étude ils choisissent, en fonction du temps que dont ils ont besoin d'apprendre à être plus souples et permettent aux étudiants d'utiliser leur temps plus efficacement encore une fois, plutôt que de l'apprentissage en classe. Avec l'enseignement à distance, les étudiants peuvent effectuer d'autres activités comme le travail, ou il est possible qu'ils explorent des sujets plus profonds qu'ils apprennent de manière plus autonome, donc indirectement encourager l'élève à être plus créatif.

\section{Conclusion}

Pour conclure, il y a du problème pour parler d'une façon naturelle chez les étudiants à la première année car il manque de temps pour s'entrainer individuellement, et aussi manque de s'exprimer en français. Cela s'explique par le trac quand ils parlent et ils utilisent la structure indonésienne. C'est la raison pour laquelle, l'entrainement hors de la classe est important, c'est le modèle de l'apprentissage à travers le Skype basé la culture. On utilise six aspects culturels ; identité, le lieu de vie, la passion et loisir, les activités de tous les jours, la famille, le voyage, la gastronomie et la mode venant après avoir analysé le manuel de l'Alter Ego+ 1 .

\section{Références}

Barreau, D. (2000). Distance Learning: Beyond Content. Journal of Education for Library and Information Science, 41, 79. https://doi.org/10.2307/40324057

Berthet, A., Hugot, C., Kizirian, V. M., Sampsonis, B., \& Waendendries, M. (2012). Alter Ego +1 Méthode de Français. Paris: Hachette.

Gall, M. D., Gall, J. P., \& Borg, W. R. (1983). Educational Research: An Introduction (4th ed.). New York: Longman.

Miarso, Y. (2011). Survei Model Pengembangan Instruksional. Jakarta: Universitas Negeri Jakarta.

Robbins, S. P. (1996). Organizational Behavior: Concepts, Controversies and Applications. New York: Prentice Hall.

Samovar, L. A., Porter, R. E., \& McDaniel, E. R. (2014). Komunikasi Lintas Budaya. Jakarta: Salemba Humanika.

Tagliante, C. (1994). La Classe de Langue. Paris : Clé International. 